\title{
An EPQ model with trapezoidal demand under volume flexibility
}

\author{
Himani Dem ${ }^{a^{*}}$, S.R. Singh ${ }^{\mathrm{b}}$ and Jitendra Kumar
}

${ }^{a}$ Department of Applied Science, Meerut Institute of Technology, Meerut-250103, India

${ }^{b}$ Department of Mathematics, Devanagri College, Chaudhary Charan Singh University, Meerut - 250001, India

${ }^{c}$ Department of Mathematics and Statistics, Banasthali University, P.O. Banasthali Vidyapith, Rajasthan - 304022, India

\section{CHRON I CLE ABSTRACT}

Article history:

Received June 22013

Received in revised format

August 152013

Accepted August 302013

Available online

September 42013

Keywords:

Production

Trapezoidal demand

Volume flexibility

\begin{abstract}
In this paper, we explored an economic production quantity model (EPQ) model for finite production rate and deteriorating items with time-dependent trapezoidal demand. The objective of the model under study is to determine the optimal production run-time as well as the number of production cycle in order to maximize the profit. Numerical example is also given to illustrate the model and sensitivity analyses regarding various parameters are performed to study their effects on the optimal policy.
\end{abstract}

\section{Introduction}

Most of the researchers considered the time varying demand as an increasing or decreasing function of time, while in practice, this assumption is not suitable for all products. The demand shows two-fold ramp type pattern for items like fashion apparel, particular kind of eatables and festival accessories have limited sales period and become obsolete at the end of period. This kind of pattern has been termed as "trapezoidal ramp-type". In the beginning of the season, the demand increases up to a certain time point and stabilizes afterwards but starts declining towards end the of the season. The economic order quantity (EOQ) model with ramp-type demand rate was initially proposed by Hill (1995). Since then several researchers and practitioners have paid significant consideration to study ramp-type demand. Mandal and Pal (1998) developed the EOQ model with ramp-type demand for exponentially deteriorating items with shortages. Wu and Ouyang (2000) investigated two inventory models assuming different replenishment policies: one started with shortage and another had shortage after inventory consumption. After that, $\mathrm{Wu}$ (2001) developed a model for deteriorating items with 
ramp-type demand and partial backlogging. Giri et al. (2003) extended ramp-type demand inventory model with more general Weibull distribution deterioration rate. Manna and Chaudhuri (2006) studied an EPQ model with ramp-type two time periods categorized demand pattern assuming demand dependent production. Deng et al. (2007) focussed on the doubtful results found by Mandal and Pal (1998) and $\mathrm{Wu}$ and Ouyang (2000) and obtained a more consistent solution. Panda et al. (2008, 2009) extended Giri et al.'s (2003) one-fold demand model to two-fold demand. Model studied by Hill (1995) was extended to trapezoidal-type demand rate by Cheng and Wang (2009). Panda et al. (2009) worked on a single-item economic production quantity (EPQ) model with quadratic ramp-type demand function in order to determine the optimal production stopping time. Model of Deng et al. (2007) was extended to more general ramp-type demand rate, Weibull distribution deterioration rate, and general partial backlogging rate by Skouri et al. (2009). Hung (2011) extended the model of Skouri et al. (2009) by applying arbitrary component in ramp-type demand pattern. Shah and Shah (2012) studied a joint vendor-buyer strategy for trapezoidal demand which is beneficial to both the players in the supply chain.

In most of the articles mentioned above, the constant rate of production is considered. But constant production rate is not always realistic. For example, when production model is based on time varying demand, the assumption of constant production rate is not suitable. Such scenarios results into application of variable production rate. The study of the model with changeable machine production rate was initiated by Schweitzer and Seidmann (1991). Khouja (1995) established a production model with unit production cost depending on used raw materials, engaged labor and tool wear and tear cost. Bhandari and Sharma (1999) measured the marketing cost in addition to generating a generalized cost function. The related studies done by Sana et al. (2007) and Sana (2010) may be noted. Dem and Singh (2012) worked on the EPQ model for damageable items with multivariate demand and volume flexibility. Dem and Singh (2013) developed an EPQ model with volume flexibility under imperfect production process. Goyal et al. (2013) developed a production model with ramp type demand and volume flexibility.

In the present paper, we develop an EPQ model for deteriorating items trapezoidal type demand rate with volume flexibility. We also assume that the inventory system includes several replenishments and all the ordering cycles are of fixed length. Such type of demand pattern is generally seen in the case of any fad or seasonal goods coming to market. The demand rate for such items increases with the time up to certain time and then stabilizes but in final phase, the demand rate decreases to a constant or zero, and then the next replenishment cycle starts. We observed that such type of demand rate is very reasonable and proposed a practical inventory replenishment policy for such type of inventory model. The remaining paper is structured as follows. In Section 2, we explain the assumptions and notation used throughout this paper. In Section 3, we formulate the mathematical model and the necessary conditions to find an optimal solution. In Section 4, we provide numerical example for each case to illustrate the model. Finally, the study is concluded in section 5.

\section{Assumptions and Notations}

\subsection{Assumptions}

1. The inventory involves single item.

2. Demand rate is dependent on time given by

$$
D(t)= \begin{cases}a+b_{1} t, & (i-1) T \leq t \leq(i-1+u) T \\ a+b_{1}(i-1+u) T, & (i-1+u) T \leq t \leq(i-1+v) T \\ a-b_{2} t, & (i-1+v) T \leq t \leq i T\end{cases}
$$

where $a, b_{1}, b_{2}>0, \mathrm{i}=1,2, \ldots, \mathrm{n}, \mathrm{u}$ and $\mathrm{v}$ are time parameters. 
The function defined above is known as trapezoidal function.

3. Production rate is $\mathrm{k}$ times demand rate, where $\mathrm{k}>1$.

4. The unit production cost is dependent on production.

5. Time horizon is finite.

6. Deterioration rate is a constant.

7. The deterioration occurs when the item is effectively in stock.

\subsection{Notations}

$D(t) \quad$ Demand rate

$P(D(t))$ Production rate, $P(D(t))=k D(t)$

$\theta \quad$ Deterioration rate

$C_{1} \quad$ Set up cost

$C_{2} \quad$ Holding cost per unit per unit time

$S_{o} \quad$ Selling price per unit

$C(P) \quad$ Production cost per unit given by $C(P)=R+\frac{G}{k D(t)}$

$R \quad$ Material cost per unit

$G \quad$ Factor associated with costs like labor and energy costs

$I(t) \quad$ Inventory level at any time $\mathrm{t}$

$T \quad$ Constant scheduling period per cycle

$t_{\mathrm{i}-1+\mathrm{u}} \quad$ Time up to which demand stabilizes and equals to $(i-1+u) T$

$t_{\mathrm{i}-1+\mathrm{v}} \quad$ Time till the demand remains stable and equals to $(i-1+v) \mathrm{T}$

$t_{\mathrm{i}-1+\mathrm{r}} \quad$ Production run time and equals to $(i-1+r) \mathrm{T}$

$n \quad$ Total number of cycles

$H \quad$ length of planning horizon and equals to $n T$

\section{Model Formulation}

We have considered the following different cases based on the occurrence of time points of demand in different phases.

3.1 Case (I) When $(i-1+v) T<(i-1+r) T$

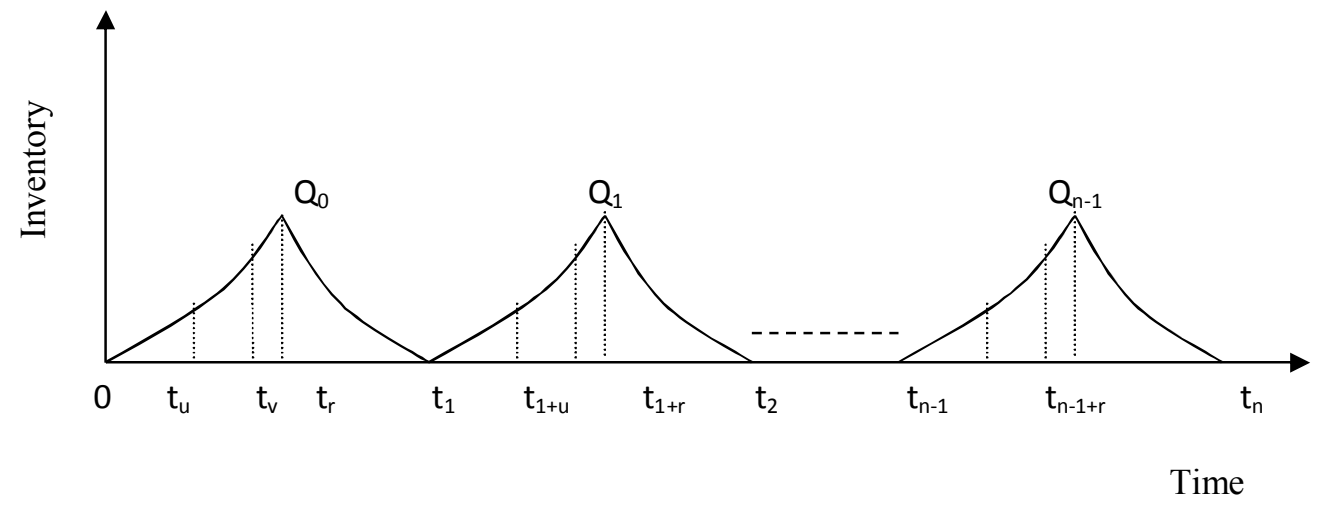

Fig.1. Graphical representation of the system for Case I

The differential equations governing the system are given as follows:

$\frac{d I_{11}(t)}{d t}=(k-1)\left(a+b_{1} t\right)-\theta I_{11}(t), \quad(i-1) T \leq t \leq(i-1+u) T, i=1,2, \ldots \ldots, n$ 


$$
\begin{array}{ll}
\frac{d I_{12}(t)}{d t}=(k-1)\left\{a+b_{1}(i-1+u) T\right\}-\theta I_{12}(t), \quad(i-1+u) T \leq t \leq(i-1+v) T \\
\frac{d I_{13}(t)}{d t}=(k-1)\left(a-b_{2} t\right)-\theta I_{13}(t), & (i-1+v) T \leq t \leq(i-1+r) T \\
\frac{d I_{2}(t)}{d t}=-\left(a-b_{2} t\right)-\theta I_{2}(t), & (i-1+r) T \leq t \leq i T
\end{array}
$$

Solving the Eq. (1) to Eq. (4) using the boundary conditions, $I_{11}((i-1) T)=0$,

$$
\begin{aligned}
& I_{12}((i-1+u) T)=I_{11}((i-1+u) T), I_{13}((i-1+v) T)=I_{12}((i-1+v) T), I_{2}(i T)=0 \\
& I_{11}(t)=-\frac{1}{\theta^{2}}\left[b_{1} k-b_{1}+\theta^{2} e^{\theta(i-1) T} e^{-\theta T}\left\{\frac{1}{\theta^{2}}\left(-b_{1} k+b_{1}\right)-\frac{1}{\theta}\left(a-a k+T b_{1}(i-1)-T b_{1} k(i-1)\right)\right\}\right]-\frac{1}{\theta}\left(a-a k+b_{1} t-b_{1} k t\right) \\
& I_{12}(t)=\frac{1}{\theta}\left[T b_{1}-a+a k-T b_{1} k-T b_{1} i-T b_{1} u+\frac{1}{\theta} e^{-\theta T} e^{\theta(i+u-1) T}\left\{b_{1}-b_{1} k\right.\right. \\
& \left.\left.+\left(b_{1} k-b_{1}+a \theta-T b_{1} \theta-a k \theta+T b_{1} k \theta+T b_{1} i-T b_{1} k i\right) e^{\theta(i-1) T} e^{-\theta(i+u-1) T}\right\}+T b_{1} k i+T b_{1} k u\right] \\
& I_{13}(t)=\frac{1}{\theta^{2}}\left(b_{2} k-b_{2}+\theta^{2} e^{\theta \theta(i+v-1) T} e^{-\theta T}\left(\frac{1}{\theta^{2}}\left(-b_{2} k+b_{2}\right)+\frac{1}{\theta}\left(a_{2}-a_{2} k+T b_{2}(k-1)(i+v-1)\right)\right.\right. \\
& \left.+\frac{1}{\theta}\left(e^{-\theta v T}(k-1)\left(T b_{1}-a+T b_{1} e^{\theta v T}-a e^{\theta_{v} T}-T b_{1} i+T b_{1} i e^{\theta v T}+T b_{1} u e^{\theta v T}\right)\right)-\frac{1}{\theta^{2}} b_{1} e^{-\theta v T}\left(e^{\theta u T}-1\right)(k-1)\right)-\frac{1}{\theta}\left(a-a k-b_{2} t+b_{2} k t\right) \\
& I_{2}(t)=-\frac{1}{\theta}\left(a-b_{2} t\right)-\frac{1}{\theta^{2}}\left(b_{2}-e^{-\theta T}\left(b_{2} e^{\theta i T}+a \theta e^{\theta i T}-T b_{2} i \theta e^{\theta i T}\right)\right)
\end{aligned}
$$

Holding cost for $i^{\text {th }}$ cycle is

$$
\begin{aligned}
& H C_{i 1}=C_{2}\left\{\int_{(i-1) T}^{(i-1+u) T} I_{11}(t) d t+\int_{(i-1+u) T}^{(i-1+v) T} I_{12}(t) d t+\int_{(i-1+v) T}^{(i-1+r) T} I_{13}(t) d t+\int_{(i-1+r) T}^{i T} I_{2}(t) d t\right\} \\
& =C_{2}\left(\frac{a}{\theta^{2}}-\frac{b_{1}}{\theta^{3}}-\frac{T b_{1}}{\theta^{2}}-\frac{a k}{\theta^{2}}+\frac{b_{1} k}{\theta^{3}}-\frac{a e^{-\theta u T}}{\theta^{2}}+\frac{b_{1} e^{-\theta u T}}{\theta^{3}}+\frac{T^{2} b_{1} u}{\theta}+\frac{T b_{1} e^{-\theta u T}}{\theta^{2}}+\frac{a k e^{-\theta u T}}{\theta^{2}}\right. \\
& -\frac{b_{1} k e^{-\theta u T}}{\theta^{3}}-\frac{T^{2} b_{1} u^{2}}{2 \theta}+\frac{T b_{1} k}{\theta^{2}}+\frac{T b_{1} i}{\theta^{2}}-\frac{T a u}{\theta}+\frac{T b_{1} u}{\theta^{2}}-\frac{T b_{1} k i}{\theta^{2}}+\frac{T a k u}{\theta}+\frac{T b_{1} k u}{\theta^{2}}-\frac{T^{2} b_{1} k u}{\theta} \\
& -\frac{T^{2} b_{1} i u}{\theta}-\frac{T b_{1} k e^{-\theta u T}}{\theta^{2}}-\frac{T b_{1} i e^{-\theta u T}}{\theta^{2}}+\frac{T^{2} b_{1} k u^{2}}{2 \theta}+\frac{T b_{1} k i e^{-\theta u T}}{\theta^{2}}+\frac{T^{2} b_{1} k i u}{\theta} \\
& +\frac{b_{1}}{\theta^{3}}-\frac{b_{1} k}{\theta^{3}}-\frac{T^{2} b_{1} u}{\theta}+\frac{T^{2} b_{1} v}{\theta}+\frac{T^{2} b_{1} u^{2}}{\theta}+\frac{T a u}{\theta}-\frac{T a v}{\theta}-\frac{b_{1}}{\theta^{3}} e^{\theta(u-v) T}-\frac{T a k u}{\theta}+\frac{T a k v}{\theta}+\frac{T^{2} b_{1} k u}{\theta} \\
& -\frac{T^{2} b_{1} k v}{\theta}+\frac{T^{2} b_{1} i u}{\theta}-\frac{T^{2} b_{1} i v}{\theta}-\frac{T^{2} b_{1} u v}{\theta}-\frac{T^{2} b_{1} k u^{2}}{\theta}+\frac{T^{2} b_{1} k u v}{\theta}-\frac{T^{2} b_{1} k i u}{\theta}+\frac{T^{2} b_{1} k i v}{\theta} \\
& +\left(\frac{a}{\theta^{2}}-\frac{b_{1}}{\theta^{3}}-\frac{T b_{1}}{\theta^{2}}-\frac{a k}{\theta^{2}}+\frac{\left(b_{1} k-b_{1}\right)}{\theta^{3}}+\frac{T b_{1} k}{\theta^{2}}+\frac{T b_{1} i}{\theta^{2}}-\frac{T b_{1} k i}{\theta^{2}}\right)\left(e^{-\theta u T}-e^{-\theta v T}\right) \\
& +\frac{b_{1}}{\theta^{3}}+\frac{T b_{1}}{\theta^{2}}+\frac{T b_{2}}{\theta^{2}}-\frac{b_{2} k}{\theta^{3}}-\frac{a}{\theta^{2}} e^{-\theta r T}+\frac{b}{\theta^{3}} e^{-\theta v T}-\frac{T^{2} b_{2} r}{\theta}+\frac{T^{2} b_{2} v}{\theta}+\left(\frac{T b_{1}}{\theta^{2}}+\frac{a k}{\theta^{2}}\right)\left(e^{-\theta r T}-e^{-\theta v T}\right) \\
& -\frac{b_{1} k}{\theta^{3}}\left(e^{-\theta r T}-e^{-\theta v T}\right)+\frac{T^{2} b_{2} r^{2}}{2 \theta}-\frac{T^{2} b_{2} v^{2}}{2 \theta}-\frac{T b_{1} k}{\theta^{2}}-\frac{T b_{2} k}{\theta^{2}}-\frac{T b_{1} i}{\theta^{2}}-\frac{T b_{2} i}{\theta^{2}}-\frac{T a r}{\theta}-\frac{T b_{2} r}{\theta^{2}}-\frac{T b_{1} u}{\theta^{2}}+\frac{T a v}{\theta} \\
& -\frac{b_{1}}{\theta^{3}} e^{\theta(u-r) T}-\frac{b_{2}}{\theta^{3}} e^{\theta(v-r) T}+\frac{b_{1}}{\theta^{3}} e^{\theta(u-v) T}+\frac{T b_{1} k i}{\theta^{2}}+\frac{T b_{2} k i}{\theta^{2}}+\frac{T a k r}{\theta}+\frac{T b_{2} k r}{\theta^{2}}+\frac{T b_{1} k u}{\theta^{2}}-\frac{T a k v}{\theta}-\frac{T b_{1}}{\theta^{2}} e^{\theta(v-r) T} \\
& +\frac{b_{1} k}{\theta^{3}} e^{\theta(u-r) T}+\frac{b_{1} k}{\theta^{3}} e^{\theta(v-r) T}-\frac{b_{1} k}{\theta^{3}} e^{\theta(u-v) T}+\frac{T^{2} b_{2} k r}{\theta}+\frac{T^{2} b_{2} i r}{\theta}-\frac{T^{2} b_{2} k v}{\theta}-\frac{T^{2} b_{2} i v}{\theta}-\frac{T b_{1} k}{\theta^{2}} e^{-\theta r T}-\frac{T b_{1} i}{\theta^{2}} e^{-\theta r T} \\
& +\frac{T b_{2} k}{\theta^{2}} e^{-\theta v T}+\frac{T b_{1} k}{\theta^{2}} e^{-\theta v T}-\frac{T^{2} b_{2} k r^{2}}{2 \theta}+\frac{T^{2} b_{2} k v^{2}}{2 \theta}+\frac{T b_{1} k i}{\theta^{2}} e^{-\theta r T}-\frac{T b_{2} k i}{\theta^{2}} e^{-\theta v T}+\frac{T b_{1} k}{\theta^{2}} e^{\theta(v-r) T}+\frac{T b_{2} k}{\theta^{2}} e^{\theta(v-r) T} \\
& +\frac{T b_{1} i}{\theta^{2}} e^{\theta(v-r) T}+\frac{T b_{2} i}{\theta^{2}} e^{\theta(v-r) T}+\frac{T b_{1} u}{\theta^{2}} e^{\theta(v-r) T}+\frac{T b_{2} v}{\theta^{2}} e^{\theta(v-r) T}-\frac{T^{2} b_{2} i k r}{\theta}+\frac{T^{2} b_{2} k i v}{\theta}-\frac{T b_{1} k i}{\theta^{2}} e^{\theta(v-r) T} \\
& -\frac{T b_{1} k u}{\theta^{2}} e^{\theta(v-r) T}-\frac{T b_{2} k v}{\theta^{2}} e^{\theta(v-r) T}+\frac{T b_{2} r}{\theta^{2}}-\frac{T^{2} b_{2} i r}{\theta}-\frac{T b_{2} i}{\theta^{2}} e^{\theta(1-r) T}+\frac{T^{2} b_{2} k i}{\theta}-\frac{b_{2}}{\theta^{3}}-\frac{T a}{\theta} \\
& \left.-\frac{T b_{2}}{\theta^{2}}-\frac{T^{2} b_{2}}{2 \theta}-\frac{a}{\theta^{2}}+\frac{T^{2} b_{2} r}{\theta}-\frac{T^{2} b_{2} r^{2}}{2 \theta}+\frac{a}{\theta^{2}} e^{\theta(1-r) T}+\frac{b_{2}}{\theta^{3}} e^{\theta(1-r) T}+\frac{T b_{2} i}{\theta^{2}}+\frac{T a r}{\theta}\right)
\end{aligned}
$$


Production cost for $i^{\text {th }}$ cycle is

$$
\begin{aligned}
P C_{i 1}= & \int_{(i-1) T}^{(i-1+u) T}\left(R+\frac{G}{k\left(a+b_{1} t\right)}\right) k\left(a+b_{1} t\right) d t+\int_{(i-1+u) T}^{(i-1+v) T}\left(R+\frac{G}{k\left\{a+b_{1}(i-1+u) T\right\}}\right) k\left\{a+b_{1}(i-1+u) T\right\} d t \\
& +\int_{(i-1+v) T}^{(i-1+r) T}\left(R+\frac{G}{k\left(a_{2}-b_{2} t\right)}\right) k\left(a_{2}-b_{2} t\right) d t \\
= & \frac{1}{2}\left(T u\left(2 G+2 R a k-2 R T b_{1} k+2 R T b_{1} k i+R T b_{1} k u\right)\right)-T(u-v)\left(G+R a k-R T b_{1} k+R T b_{1} k i+R T b_{1} k u\right) \\
+ & \frac{1}{2}\left(T(r-v)\left(2 G+2 R a k+2 R T b_{2} k-2 R T b_{2} k i-R T b_{2} k r-R T b_{2} k v\right)\right)
\end{aligned}
$$

Sales revenue for $i^{\text {th }}$ cycle is

$$
\begin{aligned}
S R_{i 1}= & S_{0}\left\{\int_{(i-1) T}^{(i-1+u) T}\left(a+b_{1} t\right) d t+\int_{(i-1+u) T}^{(i-1+v) T}\left(a+b_{1}(i-1+u) T\right) d t+\int_{(i-1+v) T}^{i T}\left(a-b_{2} t\right) d t\right\} \\
= & \frac{1}{2}\left(T S_{0} u\left(2 a-2 T b_{1}+2 T b_{1} i+T b_{1} u\right)\right)-T S_{0}(u-v)\left(a-T b_{1}+T b_{1} i+T b_{1} u\right) \\
& -\frac{1}{2}\left(T S_{0}(v-1)\left(2 a+T b_{2}-2 T b_{2} i-T b_{2} v\right)\right)
\end{aligned}
$$

Total profit per unit time of the system is

$T P_{1}=\frac{1}{H} \sum_{i}\left(S R_{i 1}-P C_{i 1}-H C_{i 1}-C_{1}\right)$

3.2 Case (II) When $(i-1+r) T<(i-1+v) T<i T$

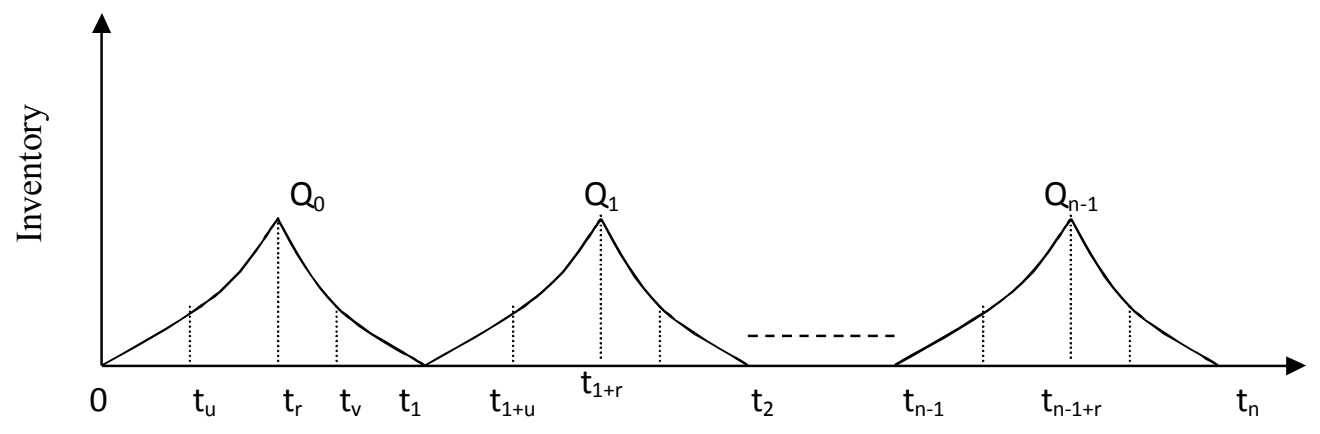

Time

Fig. 2. Graphical representation of the system for Case II

The differential equations governing the system are given as follows:

$$
\begin{aligned}
& \frac{d I_{11}(t)}{d t}=(k-1)\left(a+b_{1} t\right)-\theta I_{11}(t), \quad(i-1) T \leq t \leq(i-1+u) T, i=1,2, \ldots \ldots, n \\
& \frac{d I_{12}(t)}{d t}=(k-1)\left\{a+b_{1}(i-1+u) T\right\}-\theta I_{12}(t), \quad(i-1+u) T \leq t \leq(i-1+r) T \\
& \frac{d I_{21}(t)}{d t}=-\left\{a+b_{1}(i-1+u) T\right\}-\theta I_{21}(t), \quad(i-1+r) T \leq t \leq(i-1+v) T
\end{aligned}
$$


$\frac{d I_{22}(t)}{d t}=-\left(a-b_{2} t\right)-\theta I_{22}(t), \quad(i-1+r) T \leq t \leq i T$

Solving the Eqs. (13-16) using the boundary conditions, $I_{11}((i-1) T)=0$,

$$
\begin{aligned}
& I_{12}((i-1+u) T)=I_{11}((i-1+u) T), I_{21}((i-1+r) T)=I_{22}((i-1+r) T), I_{22}(i T)=0 \\
& I_{11}(t)=-\frac{1}{\theta^{2}}\left[b_{1} k-b_{1}+\theta^{2} e^{\theta(i-1) T} e^{-\theta T}\left\{\frac{1}{\theta^{2}}\left(-b_{1} k+b_{1}\right)-\frac{1}{\theta}\left(a-a k+T b_{1}(i-1)-T b_{1} k(i-1)\right)\right\}\right] \\
& -\frac{1}{\theta}\left(a-a k+b_{1} t-b_{1} k t\right) \\
& I_{12}(t)=\frac{1}{\theta}\left[T b_{1}-a+a k-T b_{1} k-T b_{1} i-T b_{1} u+\frac{1}{\theta} e^{-\theta T} e^{\theta(i+u-1) T}\left\{b_{1}-b_{1} k\right.\right. \\
& \left.\left.+\left(b_{1} k-b_{1}+a \theta-T b_{1} \theta-a k \theta+T b_{1} k \theta+T b_{1} i-T b_{1} k i\right) e^{\theta(i-1) T} e^{-\theta(i+u-1) T}\right\}+T b_{1} k i+T b_{1} k u\right] \\
& I_{21}(t)=-\frac{1}{\theta}\left(-T b_{1}+a+T b_{1} i+T b_{1} u-\frac{1}{\theta}\left(e ^ { - \theta T } e ^ { \theta ( i + r - 1 ) T } \left(b_{1} e^{-\theta(i+r-1) T} e^{\theta(i+u-1) T}+a k \theta-b_{1} e^{\theta(i-1) T} e^{-\theta(i+r-1) T}\right.\right.\right. \\
& +b_{1} k e^{\theta(i-1) T} e^{-\theta(i+r-1) T}+a \theta e^{\theta(i-1) T} e^{-\theta(i+r-1) T}-b_{1} k e^{\theta(i+u-1) T-\theta(i+r-1) T}-T b_{1} k \theta+T b_{1} k i \theta+T b_{1} k \theta u \\
& -T b_{1} \theta e^{\theta(i-1) T-\theta(i+r-1) T}-a k \theta e^{\theta(i-1) T} e^{-\theta(i+r-1) T}+T b_{1} k \theta e^{\theta(i-1) T-\theta(i+r-1) T}+T b_{1} i \theta e^{\theta(i-1) T-\theta(i+r-1) T} \\
& \left.\left.\left.-T b_{1} k i \theta e^{\theta(i-1) T-\theta(i+r-1) T}\right)\right)\right) \\
& I_{22}(t)=-\frac{1}{\theta}\left(a-b_{2} t\right)-\frac{1}{\theta^{2}}\left(b_{2}-e^{-\theta T}\left(b_{2} e^{\theta i T}+a \theta e^{\theta i T}-T b_{2} i \theta e^{\theta i T}\right)\right)
\end{aligned}
$$

Holding cost for $i^{\text {th }}$ cycle is

$$
\begin{aligned}
& H C_{i 2}=C_{2}\left\{\int_{(i-1) T}^{(i-1+u) T} I_{11}(t) d t+\int_{(i-1+u) T}^{(i-1+r) T} I_{12}(t) d t+\int_{(i-1+r) T}^{(i-1+v) T} I_{21}(t) d t+\int_{(i-1+v) T}^{i T} I_{22}(t) d t\right\} \\
& =C_{2}\left(\frac{a}{\theta^{2}}-\frac{b_{1}}{\theta^{3}}-\frac{T b_{1}}{\theta^{2}}-\frac{a k}{\theta^{2}}+\frac{b_{1} k}{\theta^{3}}-\frac{a e^{-\theta u T}}{\theta^{2}}+\frac{b_{1} e^{-\theta u T}}{\theta^{3}}+\frac{T^{2} b_{1} u}{\theta}+\frac{T b_{1} e^{-\theta u T}}{\theta^{2}}+\frac{a k e^{-\theta u T}}{\theta^{2}}-\frac{b_{1} k e^{-\theta u T}}{\theta^{3}}-\frac{T^{2} b_{1} u^{2}}{2 \theta}\right. \\
& +\frac{T b_{1} k}{\theta^{2}}+\frac{T b_{1} i}{\theta^{2}}-\frac{T a u}{\theta}+\frac{T b_{1} u}{\theta^{2}}-\frac{T b_{1} k i}{\theta^{2}}+\frac{T a k u}{\theta}+\frac{T b_{1} k u}{\theta^{2}}-\frac{T^{2} b_{1} k u}{\theta}-\frac{T^{2} b_{1} i u}{\theta}-\frac{T b_{1} k e^{-\theta u T}}{\theta^{2}}-\frac{T b_{1} i e^{-\theta u T}}{\theta^{2}}+\frac{T^{2} b_{1} k u^{2}}{2 \theta} \\
& +\frac{T b_{1} k i e^{-\theta u T}}{\theta^{2}}+\frac{T^{2} b_{1} k i u}{\theta}+\frac{b_{1}}{\theta^{3}}-\frac{b_{1} k}{\theta^{3}}+\left(-\frac{a}{\theta^{2}}+\frac{b_{1}}{\theta^{3}}+\frac{T b_{1}}{\theta^{2}}+\frac{a k}{\theta^{2}}-\frac{b_{1} k}{\theta^{3}}\right)\left(e^{-\theta r T}-e^{-\theta u T}\right)-\frac{T^{2} b_{1} u}{\theta}+\frac{T^{2} b_{1} r}{\theta}+\frac{T^{2} b_{1} u^{2}}{\theta} \\
& +\frac{T a u}{\theta}-\frac{T a r}{\theta}-\frac{b_{1}}{\theta^{3}} e^{\theta(u-r) T}-\frac{T a k u}{\theta}+\frac{T a k r}{\theta}+\frac{b_{1} k}{\theta^{3}} e^{\theta(u-r) T}+\frac{T^{2} b_{1} k u}{\theta}-\frac{T^{2} b_{1} k r}{\theta}+\frac{T^{2} b_{1} i u}{\theta}-\frac{T^{2} b_{1} i r}{\theta} \\
& -\frac{T^{2} b_{1} r u}{\theta}-\frac{T b_{1} k}{\theta^{2}} e^{-\theta r T}-\frac{T b_{1} i}{\theta^{2}} e^{-\theta r T}+\frac{T b_{1} k}{\theta^{2}} e^{-\theta u T}+\frac{T b_{1} i}{\theta^{2}} e^{-\theta u T}-\frac{T^{2} b_{1} k u^{2}}{\theta}+\frac{T b_{1} k i}{\theta^{2}}\left(e^{-\theta r T}-e^{-\theta u T}\right) \\
& +\frac{T^{2} b_{1} k i r}{\theta}-\frac{T^{2} b_{1} k i u}{\theta}+\frac{T^{2} b_{1} k r u}{\theta}+\frac{a k}{\theta^{2}}+\left(\frac{a}{\theta^{2}}-\frac{b_{1}}{\theta^{3}}-\frac{T b_{1}}{\theta^{2}}-\frac{a k}{\theta^{2}}+\frac{b_{1} k}{\theta^{3}}+\frac{T b_{1} k}{\theta^{2}}+\frac{T b_{1} i}{\theta^{2}}-\frac{T b_{1} k i}{\theta^{2}}\right)\left(e^{-\theta r T}-e^{-\theta v T}\right) \\
& -\frac{T^{2} b_{1} r}{\theta}+\frac{T^{2} b_{1} v}{\theta}-\frac{T b_{1} k}{\theta^{2}}+\frac{T a r}{\theta^{2}}-\frac{T a v}{\theta^{2}}+\frac{b_{1} k}{\theta^{3}} e^{\theta(u-r) T}+\frac{b_{1} k}{\theta^{3}} e^{\theta(u-v) T}+\frac{T^{2} b_{1} i r}{\theta}-\frac{T^{2} b_{1} i v}{\theta}+\frac{T^{2} b_{1} r u}{\theta}-\frac{T^{2} b_{1} u v}{\theta} \\
& +\frac{T b_{1} k}{\theta^{2}} e^{\theta(v-r) T}-\frac{T b_{1} k i}{\theta^{2}} e^{\theta(v-r) T}-\frac{T b_{1} k u}{\theta^{2}} e^{\theta(v-r) T}+\frac{T^{2} b_{2} i}{\theta}-\frac{b_{2}}{\theta^{3}}-\frac{T a}{\theta}-\frac{T b_{2}}{\theta^{2}}-\frac{T^{2} b_{2}}{2 \theta}-\frac{a}{\theta^{2}}+\frac{T^{2} b_{2} v}{\theta}-\frac{T^{2} b_{2} v^{2}}{2 \theta} \\
& \left.+\left(\frac{a}{\theta^{2}}+\frac{b_{2}}{\theta^{3}}-\frac{T b_{2} i}{\theta^{2}}\right) e^{-\theta(v-1) T}+\frac{T b_{2} i}{\theta^{2}}+\frac{T a v}{\theta}+\frac{T b_{2} v}{\theta^{2}}-\frac{T b_{2} i v}{\theta}\right)
\end{aligned}
$$


Production cost for $i^{\text {th }}$ cycle is

$$
\begin{aligned}
& P C_{i 2}=\int_{(i-1) T}^{(i-1+u) T}\left(R+\frac{G}{k\left(a+b_{1} t\right)}\right) k\left(a+b_{1} t\right) d t+\int_{(i-1+u) T}^{(i-1+r) T}\left(R+\frac{G}{k\left\{a+b_{1}(i-1+u) T\right\}}\right) k\left\{a+b_{1}(i-1+u) T\right\} d t \\
& =\frac{1}{2}\left(T u\left(2 G+2 R a k-2 R T b_{1} k+2 R T b_{1} k i+R T b_{1} k u\right)\right)+T(r-u)\left(G+R a k-R T b_{1} k+R T b_{1} k i+R T b_{1} k u\right)
\end{aligned}
$$

Sales revenue for $i^{\text {th }}$ cycle is

$$
\begin{aligned}
& S R_{i 2}=S_{0}\left\{\int_{(i-1) T}^{(i-1+u) T}\left(a+b_{1} t\right) d t+\int_{(i-1+u) T}^{(i-1+v) T}\left(a+b_{1}(i-1+u) T\right) d t+\int_{(i-1+v) T}^{i T}\left(a-b_{2} t\right) d t\right\} \\
& =\frac{1}{2}\left(T S_{0} u\left(2 a-2 T b_{1}+2 T b_{1} i+T b_{1} u\right)\right)-T S_{0}(u-v)\left(a-T b_{1}+T b_{1} i+T b_{1} u\right) \\
& -\frac{1}{2}\left(T S_{0}(v-1)\left(2 a+T b_{2}-2 T b_{2} i-T b_{2} v\right)\right)
\end{aligned}
$$

Total profit per unit time of the system is

$$
T P_{2}=\frac{1}{H} \sum_{i}\left(S R_{i 2}-P C_{i 2}-H C_{i 2}-C_{1}\right)
$$

3.3 Case (III) When $(i-1+r) T<(i-1+u) T<i T$

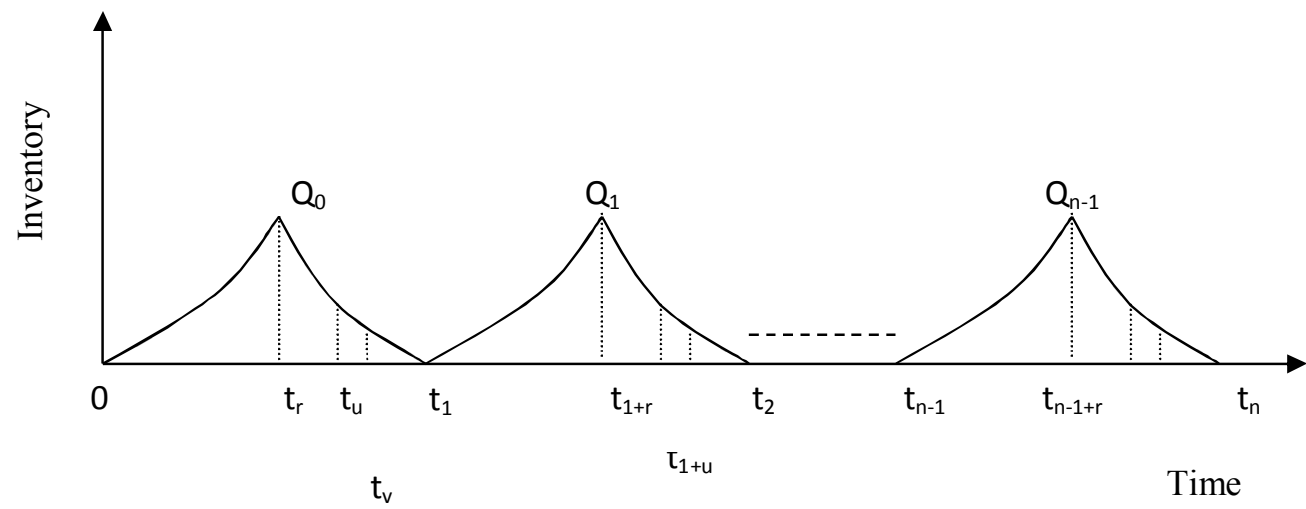

Fig. 3. Graphical representation of the system for Case III

The differential equations governing the system are given as follows:

$$
\begin{array}{ll}
\frac{d I_{11}(t)}{d t}=(k-1)\left(a+b_{1} t\right)-\theta I_{11}(t), & (i-1) T \leq t \leq(i-1+r) T, i=1,2, \ldots \ldots, n \\
\frac{d I_{21}(t)}{d t}=-\left(a+b_{1} t\right)-\theta I_{21}(t), & (i-1+r) T \leq t \leq(i-1+u) T \\
\frac{d I_{22}(t)}{d t}=-\left\{a+b_{1}(i-1+u) T\right\}-\theta I_{22}(t), & (i-1+u) T \leq t \leq(i-1+v) T \\
\frac{d I_{23}(t)}{d t}=-\left(a-b_{2} t\right)-\theta I_{23}(t), & (i-1+v) T \leq t \leq i T
\end{array}
$$

Solving the Eqs. (25-28) using the boundary conditions,

$$
\begin{aligned}
& I_{11}((i-1) T)=0, I_{21}((i-1+r) T)=I_{1}((i-1+r) T), I_{21}((i-1+u) T)=I_{22}((i-1+u) T), I_{23}(i T)=0 \\
& I_{11}(t)=-\frac{1}{\theta^{2}}\left[b_{1} k-b_{1}+\theta^{2} e^{\theta(i-1) T} e^{-\theta T}\left\{\frac{1}{\theta^{2}}\left(-b_{1} k+b_{1}\right)-\frac{1}{\theta}\left(a-a k+T b_{1}(i-1)-T b_{1} k(i-1)\right)\right\}\right]-\frac{1}{\theta}\left(a-a k+b_{1} t-b_{1} k t\right)
\end{aligned}
$$




$$
\begin{aligned}
& I_{21}(t)=\frac{1}{\theta^{2}}\left(b_{1}-\theta^{2} e^{-\theta T} e^{\theta(i+r-1) T}\left(\frac{1}{\theta}\left(a-a k+T b_{1}(i+r-1)-T b_{1} k(i+r-1)\right)-\frac{1}{\theta}\left(a+T b_{1}(i+r-1)\right)+\frac{b}{\theta^{2}}\right.\right. \\
& \left.\left.+\frac{1}{\theta^{2}}\left(b_{1} k-b_{1}+\theta^{2} e^{\theta(i-1) T} e^{\theta(i+r-1) T}\left(\frac{1}{\theta^{2}}\left(-b_{1} k+b_{1}\right)-\frac{1}{\theta}\left(a-a k+T b_{1}(i-1)-T b_{1} k(i-1)\right)\right)\right)\right)\right)-\frac{1}{\theta}\left(a+b_{1} t\right) \\
& I_{22}(t)=-\frac{1}{\theta}\left(a-T b_{1}+T b_{1} i+T b_{1} u-\frac{1}{\theta}\left(e ^ { - \theta T } e ^ { \theta ( i + u - 1 ) T } \left(b_{1}+\left(-b_{1}+b_{1} k+a \theta-T b_{1}-a k \theta+T b_{1} k+T b_{1} i\right.\right.\right.\right. \\
& \left.\left.\left.\left.-T b_{1} k i \theta\right) e^{\theta(i-1) T} e^{-\theta(i+u-1) T}+\left(b_{1} k+a k \theta-T b_{1} k \theta+T b_{1} k r \theta+T b_{1} k i \theta\right) e^{\theta(i+r-1) T} e^{-\theta(i+u-1) T}\right)\right)\right) \\
& I_{23}(t)=-\frac{1}{\theta}\left(a-b_{2} t\right)-\frac{1}{\theta^{2}}\left(b_{2}-e^{-\theta T}\left(b_{2} e^{\theta i T}+a \theta e^{\theta i T}-T b_{2} i \theta e^{\theta i T}\right)\right)
\end{aligned}
$$

Holding cost for $i^{\text {th }}$ cycle is

$$
\begin{aligned}
& H C_{i 3}=C_{2}\left\{\int_{(i-1) T}^{(i-1+r) T} I_{1}(t) d t+\int_{(i-1+r) T}^{(i-1+u) T} I_{21}(t) d t+\int_{(i-1+u) T}^{(i-1+v) T} I_{22}(t) d t+\int_{(i-1+v) T}^{i T} I_{23}(t) d t\right\} \\
& =C_{2}\left(\frac{a}{\theta^{2}}-\frac{b_{1}}{\theta^{3}}-\frac{T b_{1}}{\theta^{2}}-\frac{a k}{\theta^{2}}+\frac{b_{1} k}{\theta^{3}}-\frac{a e^{-\theta u T}}{\theta^{2}}+\frac{b_{1} e^{-\theta u T}}{\theta^{3}}+\frac{T^{2} b_{1} u}{\theta}+\frac{T b_{1} e^{-\theta u T}}{\theta^{2}}+\frac{a k e^{-\theta u T}}{\theta^{2}}\right. \\
& -\frac{b_{1} k e^{-\theta u T}}{\theta^{3}}-\frac{T^{2} b_{1} u^{2}}{2 \theta}+\frac{T b_{1} k}{\theta^{2}}+\frac{T b_{1} i}{\theta^{2}}-\frac{T a u}{\theta}+\frac{T b_{1} u}{\theta^{2}}-\frac{T b_{1} k i}{\theta^{2}}+\frac{T a k u}{\theta}+\frac{T b_{1} k u}{\theta^{2}}-\frac{T^{2} b_{1} k u}{\theta} \\
& -\frac{T^{2} b_{1} i u}{\theta}-\frac{T b_{1} k e^{-\theta u T}}{\theta^{2}}-\frac{T b_{1} i e^{-\theta u T}}{\theta^{2}}+\frac{T^{2} b_{1} k u^{2}}{2 \theta}+\frac{T b_{1} k i e^{-\theta u T}}{\theta^{2}}+\frac{T^{2} b_{1} k i u}{\theta} \\
& +\frac{a k}{\theta^{2}}-\frac{b_{1} k}{\theta^{3}}+\left(\frac{a}{\theta^{2}}-\frac{b_{1}}{\theta^{3}}-\frac{T b_{1}}{\theta^{2}}-\frac{a k}{\theta^{2}}+\frac{b_{1} k}{\theta^{3}}+\frac{T b_{1} k i}{\theta^{2}}-\frac{T b_{1} k}{\theta^{2}}+\frac{T b_{1} i}{\theta^{2}}\right)\left(e^{-\theta r T}-e^{-\theta u T}\right)-\frac{T^{2} b_{1} r}{\theta}+\frac{T^{2} b_{1} u}{\theta}+\frac{T^{2} b_{1} r^{2}}{2 \theta}-\frac{T^{2} b_{1} u^{2}}{2 \theta} \\
& -\frac{T b_{1} k}{\theta^{2}}+\frac{T a r}{\theta}-\frac{T b_{1} r}{\theta^{2}}-\frac{T a u}{\theta}+\frac{T b_{1} u}{\theta^{2}}+\frac{T b_{1} k i}{\theta^{2}}+\frac{T b_{1} k r}{\theta^{2}}-\frac{a k}{\theta^{2}} e^{-\theta(u-r) T}+\frac{b_{1} k}{\theta^{3}} e^{-\theta(u-r) T}+\frac{T^{2} b_{1} i r}{\theta}-\frac{T^{2} b_{1} i u}{\theta} \\
& -\frac{T b_{1} k i}{\theta^{2}} e^{-\theta(u-r) T}-\frac{T b_{1} k}{\theta^{2}} e^{-\theta(u-r) T}+\frac{b_{1}}{\theta^{3}}+\left(\frac{a}{\theta^{2}}-\frac{b_{1}}{\theta^{3}}-\frac{T b_{1}}{\theta^{2}}-\frac{a k}{\theta^{2}}+\frac{b_{1} k}{\theta^{3}}+\frac{T b_{1} k}{\theta^{2}}+\frac{T b_{1} i}{\theta^{2}}-\frac{T b_{1} k i}{\theta^{2}}\right)\left(e^{-\theta u T}-e^{-\theta v T}\right) \\
& -\frac{T^{2} b_{1} u}{\theta}+\frac{T^{2} b_{1} v}{\theta}+\frac{T^{2} b_{1} u^{2}}{\theta}+\frac{T a u}{\theta}-\frac{T a v}{\theta}-\frac{b_{1}}{\theta^{3}} e^{-\theta(u-v) T}+\frac{a k}{\theta^{2}} e^{-\theta(u-r) T}-\frac{a k}{\theta^{2}} e^{-\theta(v-r) T}-\frac{b_{1} k}{\theta^{3}} e^{-\theta(u-r) T} \\
& +\frac{b_{1} k}{\theta^{3}} e^{-\theta(v-r) T}+\frac{T^{2} b_{1} i u}{\theta}-\frac{T^{2} b_{1} i v}{\theta}-\frac{T^{2} b_{1} u v}{\theta}-\frac{T b_{1} k}{\theta^{2}} e^{-\theta(u-r) T}+\frac{T b_{1} k}{\theta^{2}} e^{-\theta(v-r) T}+\frac{T b_{1} k i}{\theta^{2}} e^{-\theta(u-r) T}-\frac{T b_{1} k i}{\theta^{2}} e^{-\theta(v-r) T} \\
& +\frac{T b_{1} k r}{\theta^{2}} e^{-\theta(u-r) T}-\frac{T b_{1} k}{\theta^{2}} e^{-\theta(v-r) T}+\frac{T^{2} b_{2} i}{\theta}-\frac{b_{2}}{\theta^{3}}-\frac{T a}{\theta}-\frac{T b_{2}}{\theta^{2}}-\frac{T^{2} b_{2}}{2 \theta}-\frac{a}{\theta^{2}}+\frac{T^{2} b_{2} v}{\theta}-\frac{T^{2} b_{2} v^{2}}{2 \theta}+\frac{a}{\theta^{2}} e^{-\theta(v-1) T} \\
& \left.+\frac{b_{2}}{\theta^{3}} e^{-\theta(v-1) T}+\frac{T b_{2} i}{\theta^{2}}+\frac{T a v}{\theta}+\frac{T b_{2} v}{\theta^{2}}-\frac{T^{2} b_{2} i v}{\theta}-\frac{T b_{2} i}{\theta^{2}} e^{-\theta(v-1) T}\right)
\end{aligned}
$$

Production cost for $i^{\text {th }}$ cycle is

$$
P C_{i 3}=\int_{(i-1) T}^{(i-1+r) T}\left(R+\frac{G}{k\left(a+b_{1} t\right)}\right) k\left(a+b_{1} t\right) d t=\frac{1}{2}(\operatorname{Tr}(2 G+2 R a k-2 R T b k+2 R T b k i+R T b k r))
$$

Sales revenue for $i^{\text {th }}$ cycle is

$$
\begin{aligned}
& S R_{i 3}=S_{0}\left\{\int_{(i-1) T}^{(i-1+u) T}\left(a+b_{1} t\right) d t+\int_{(i-1+u) T}^{(i-1+v) T}\left(a+b_{1}(i-1+u) T\right) d t+\int_{(i-1+v) T}^{i T}\left(a-b_{2} t\right) d t\right\} \\
& =\frac{1}{2}\left(T S_{0} u\left(2 a-2 T b_{1}+2 T b_{1} i+T b_{1} u\right)\right)-T S_{0}(u-v)\left(a-T b_{1}+T b_{1} i+T b_{1} u\right) \\
& -\frac{1}{2}\left(T S_{0}(v-1)\left(2 a+T b_{2}-2 T b_{2} i-T b_{2} v\right)\right)
\end{aligned}
$$


Total profit per unit time of the system is

$T P_{3}=\frac{1}{H} \sum_{i}\left(S R_{i 3}-P C_{i 3}-H C_{i 3}-C_{1}\right)$

Our objective is to find maximum total profit per unit time in each case, i.e., $\max T P_{m}(n, r)=\frac{1}{H} \sum_{i}\left(S R_{i m}-P C_{i m}-H C_{i m}-C_{1}\right)$, where $\mathrm{n}$ is a positive integer and $0<r<1, m=1,2,3$

\section{Solution Procedure}

The solution procedure is as follows:

Step 1. Let $n$ be a fixed positive integer.

Step 2. Equate the first derivatives of $T P_{m}$ in Eq. (12), Eq. (24) and Eq. (36), denoted by $T P_{m}(\mathrm{r} \mid \mathrm{n})$ with respect to $r$ to zero and solve all the three equations for $r$.

Step 3. Check for concavity. The sufficient condition for maximum $T P_{m}(\mathrm{r} \mid \mathrm{n})$ is $\frac{d^{2} T P_{m}(r \mid n)}{d r^{2}}<0$.

Step 4. If $0 \leq r \leq 1$, calculate $T P_{m}$ from Eq. (12), Eq. (24) and Eq. (36).

Step 5. Repeat Step 2 to Step 4 by assuming different positive integer values of $n$. The optimal solution, $\left(n^{*}, r\right)$ must satisfy the following condition:

$T P_{m}\left(n^{*}-1, r\right) \leq T P_{m}\left(n^{*}, r\right) \geq T P_{m}\left(n^{*}+1, r\right)$

\section{Numerical Example and Sensitivity Analysis}

We determine the optimal value of decision variables and net profit using solution procedure defined in last section. We use the following values of the parameters in appropriate units: $\mathrm{S}_{0}=10, \mathrm{~T}=6, \mathrm{k}=1.5$, $\mathrm{C}_{1}=100, \mathrm{C}_{2}=1, a=10, \mathrm{~b}_{1}=\mathrm{b}_{2}=0.1, \theta=0.01$. The optimal net profit $T P_{m}{ }^{*}$, optimal number of cycles $n$ and optimal time parameter for production run time $r^{*}$ for the three cases are provided in Table 1 .

Table 1

Summary of results

\begin{tabular}{cccc}
\hline Case & $\boldsymbol{n}^{*}$ & $\boldsymbol{r}^{*}$ & $\boldsymbol{T P}_{\boldsymbol{m}}{ }^{*}$ \\
\hline I & 6 & 0.1065 & 1539 \\
II & 6 & 0.9585 & 688.90 \\
III & 6 & 0.9585 & 718.10 \\
\hline
\end{tabular}

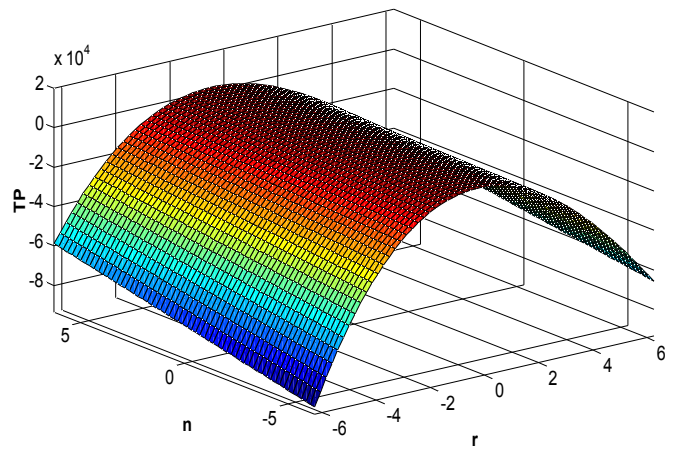

Fig. 4. Net profit in Case (I)

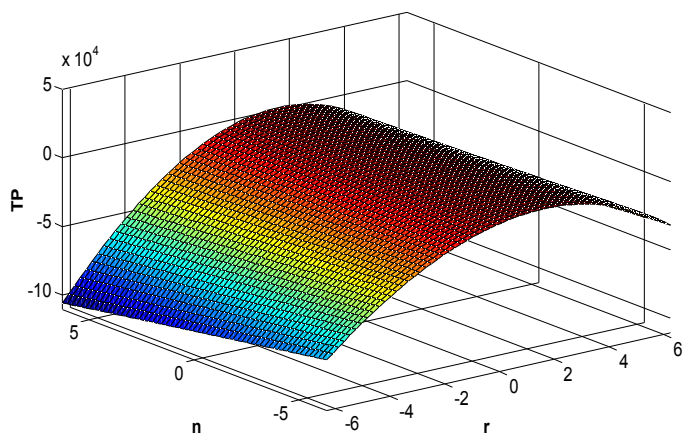

Fig. 5. Net profit in Case (II) 


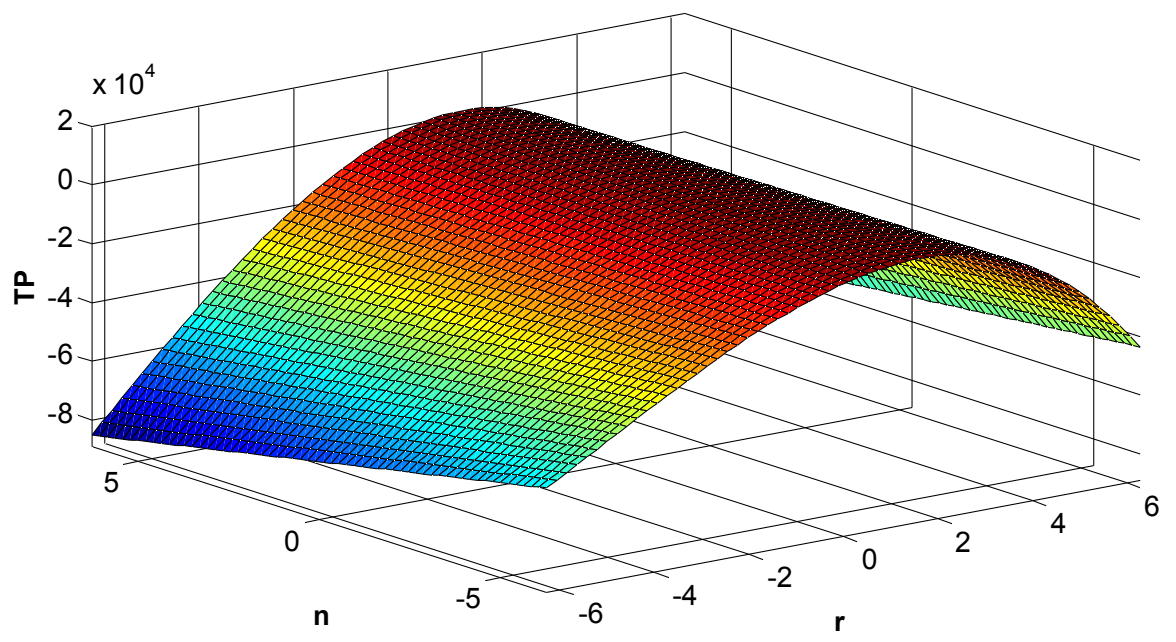

Fig. 6. Net profit in Case (III)

Keeping in view the above experimental results, sensitivity analysis is performed for Case I in which the maximum profit is obtained. It is carried out by changing the parameters by $50 \%$ and $25 \%$, taking one parameter at a time and keeping all other parameters fixed. Change in percentage in total cost of the system, denoted by PCT, corresponding to various parameters is calculated and specified in last column of each of the tables given below.

Table 2

Sensitivity analyses

\begin{tabular}{|c|c|c|c|c|c|}
\hline Parameter & Value & $T P_{m}$ & Parameter & Value & $T P_{m}$ \\
\hline \multirow{4}{*}{$S_{o}$} & 5 & -298.40 & \multirow{4}{*}{$a$} & 5 & 1212 \\
\hline & 7.5 & 620.20 & & 7.5 & 1379 \\
\hline & 12.5 & 2457 & & 12.5 & 1705 \\
\hline & 15 & 3385 & & 15 & 1872 \\
\hline \multirow{4}{*}{$b_{1}$} & 0.05 & 1481 & \multirow{4}{*}{$k$} & 0.75 & 1880 \\
\hline & 0.075 & 1526 & & 1.125 & 1713 \\
\hline & 0.125 & 1568 & & 1.875 & 1368 \\
\hline & 0.15 & 1596 & & 2.25 & 1197 \\
\hline \multirow{4}{*}{$\theta$} & 0.005 & 1545 & \multirow{4}{*}{$u$} & 0.15 & 1532 \\
\hline & 0.0075 & 1549 & & 0.225 & 1537 \\
\hline & 0.0125 & 1536 & & 0.375 & 1547 \\
\hline & 0.015 & 1533 & & 0.45 & 1551 \\
\hline \multirow{4}{*}{$v$} & 0.3 & 1400 & \multirow{4}{*}{$R$} & 2.5 & 1939 \\
\hline & 0.45 & 1474 & & 3.75 & 1740 \\
\hline & 0.75 & 1625 & & 6.25 & 1337 \\
\hline & 0.9 & 1722 & & 7.5 & 1152 \\
\hline \multirow{4}{*}{$G$} & 9 & 1573 & & & \\
\hline & 13.5 & 1556 & & & \\
\hline & 22.5 & 1528 & & & \\
\hline & 27 & 1518 & & & \\
\hline
\end{tabular}

The effect of various parameters on total profit is listed in tabular form (see, Table 2). The observations are as follows: 
1. Total profit increases with the increasing selling price $S_{o}$, which is a quite expected phenomenon.

2. Total profit increases with the increasing initial parameter ' $a$ ' and time sensitive parameter ' $b_{1}$ ' of demand. High demand of manufactured units motivates more production which in turn becomes a significant reason to earn extra profit.

3. As the coefficient related to production rate ' $k$ ' increases, total profit decreases. For higher values of ' $k$ ', quantity of production becomes more in proportion to the demand.

4. Total profit decreases with increasing value of the deterioration rate ' $\theta$ '. Because the greater amount of deteriorated units results into loss of sales that could have been contributed to profit.

5. As the parameters ' $u$ ' and ' $v$ ' increase, total profit increases. Since, ' $u$ ' contributes to the point up to which demand stabilizes and ' $v$ ' contributes to the point till the demand remains stable, therefore, their increment results into greater demand which causes greater profit.

6. The value of profit function decreases with the increasing value of parameters ' $R$ ' and ' $G$ ' and ' $J$ '. It is suitable that the unit production cost increases with the increasing material cost, labor cost and tool/die cost. If unit production cost becomes more than selling price, the total profit turns out to be negative. This is a disagreeable condition for any business policy whereas less unit production cost is always acceptable.

\section{Conclusion}

In this paper, particular items following trapezoidal ramp type demand has been considered. Three cases according to the demand has been focused and for each case, a profit function is formulated, which is to be maximized. The condition to find the optimal solution is provided and using numerical experiment, we observe that the profit is maximum when the demand starts declining before the production stops. Sensitivity analysis is performed corresponding to various parameters and the results show that our mathematical model is more realistic. The proposed model can be further enriched in several ways like stochastic or fuzzy modelling.

\section{References}

Bhandari, R.M., \& Sharma, P.K. (1999). The economic production lot size model with variable cost function. Opsearch, 36, 137- 150.

Cheng, M. \& Wang, G. (2009). A note on the inventory model for deteriorating items with trapezoidal type demand rate. Computers and Industrial Engineering, 56, 1296-1300.

Dem, H. \& Singh, S.R. (2012). Production scheduling for damageable items with demand and cost flexibility using genetic algorithm. Advances in Intelligent and Soft Computing, 131, 747-759.

Dem, H. \& Singh, S.R. (2013). A production model for ameliorating items with quality consideration. International Journal of Operational Research, 17 183-198.

Deng, P. S., Lin, R. H. J. \& Chu, P. (2007). A note on the inventory models for deteriorating items with ramp type demand rate. European Journal of Operational Research, 178, 112-120.

Goyal, S.K., Singh, S.R. \& Dem, H. (2013). Production policy for ameliorating/deteriorating items with ramp type demand. International Journal of Procurement Management, 6, 444-465.

Giri, B. C., Jalan, A. K. \& Chaudhuri, K. S. (2003). Economic Order Quantity model withWeibull deterioration distribution, shortage and ramp-type demand. International Journal of Systems Science, 34, 237-243.

Hill, R.M. (1995). Inventory models for increasing demand followed by level demand. Journal of the Operational Research Society, 46, 1250-1259.

Hung, K. C. (2011). An inventory model with generalized type demand, deterioration and backorder rates. European Journal of Operational Research, 208, 239-242.

Mandal, B. \& Pal, A. K. (1998). Order level inventory system with ramp type demand rate for deteriorating items. Journal of Interdisciplinary Mathematics, 1, 49-66. 
Manna, S. K. \& Chaudhuri, K. S. (2006). An EOQ model with ramp type demand rate, time dependent deterioration rate, unit production cost and shortages. European Journal of Operational Research, 171, 557-566.

Panda, S., Saha, S. \& Basu, M. (2007). An EOQ model with generalized ramp-type demand andweibull distribution deterioration. Asia-Pacific Journal of Operational Research, 24, 93-109.

Panda, S., Senapati, S. \& Basu, M. (2008). Optimal replenishment policy for perishable seasonal products in a season with ramp type time dependent demand. Computers and Industrial Engineering, 54, 301-314.

Panda, S., Senapati, S. \& Basu, M. (2009). Optimal production stopping time for perishable products with ramp-type quadratic demand dependent production and setup cost. Central European Journal of Operations Research, 17, 381-396.

Sana, S.S., Goyal, S.K. \& Chaudhuri, K.S. (2007). An imperfect production process in a volume flexible inventory model. International Journal of Production Economics, 105, 548-559.

Sana, S.S. (2010). A production-inventory model in an imperfect production process. European Journal of Operational Research, 200, 451-464.

Schweitzer, P.J. \& Seidmann, A. (1991). Optimizing processing rates for flexible manufacturing systems. Management Science, 37, 454-466.

Shah, N.H. \& Shah, D.B. (2012). Vendor-buyer ordering policy when demand is trapezoidal. International Journal of Industrial Engineering Computations, 3, 721-730.

Skouri, K., Konstantaras, I., Papachristos, S. \& Ganas, I. (2009). Inventory models with ramp type demand rate, partial backlogging and Weibull deterioration rate. European Journal of Operational Research, 192, 79-92.

Wu, K. S. \& Ouyang, L. Y. (2000). A replenishment policy for deteriorating items with ramp type demand rate. Proceedings of the National Science Council, Republic of China A, 24, 279-286.

$\mathrm{Wu}, \mathrm{K}$. S. (2001). An EOQ inventory model for items with Weibull distribution deterioration, ramp type demand rate and partial backlogging. Production Planning and Control, 12, 787-793. 